\title{
Efecto de la suplementación con ciclodextrinas cargadas con colesterol al semen sexado bovino post-descongelación para su uso en producción in vitro de embriones
}

\author{
Ferré, L. ${ }^{\circledR} ;$ Grötter, L. ${ }^{2}$; Fresno, C. ${ }^{3}$ y Cattaneo, L. ${ }^{2}$
}

IINTA Rafaela, Santa Fe. Argentina.

2Facultad Ciencias Veterinarias, UNL, Santa Fe, Argentina.

${ }^{3}$ Grupo de Genómica Poblacional y Bioinformática, Instituto Nacional de Medicina Genómica, México.

\section{Palabras ClaVe ADICIONALES}

Fertilización in vitro.

Espermatozoide.

Clivaje.

Blastocisto.

\section{RESUMEN}

Los espermatozoides bovinos presentan una bajo relación de colesterol/fosfolípidos en la membrana plasmática. Esta característica es importante ya que el colesterol juega un papel fundamental en el mantenimiento de la estructura y funcionalidad de la membrana espermática tras la congelación, y, por lo tanto, en la supervivencia del espermatozoide tras el proceso de criopreservación. El proceso de sexado podría agravar todavía aún más esta situación. El objetivo de este trabajo es estabilizar las membranas plasmáticas de espermatozoides sexados mediante el agregado de ciclodextrinas cargadas con colesterol (CLC) post-descongelado y consecuentemente, prolongar su funcionalidad, aumentar su capacidad de fecundar ovocitos in vitro y producción de embriones. Para ello se trataron espermatozoides sexados con $\mathrm{CLC}$ a diferentes concentraciones e incubados por 15 minutos antes de evaluar el patrón de motilidad mediante un sistema computarizado CASA y fertilizar ovocitos in vitro. Los espermatozoides tratados con $3 \mathrm{mg}$ de metil-ß-ciclodextrina saturada de colesterol cada $120 \times 10^{6}$ espermatozoides presentaron mejor motilidad y vigor y se mantuvieron elevados durante el tiempo analizado. La concentración de $3 \mathrm{mg}$ de CLC también produjo mayor clivaje, MOR/eBL, BL y embriones totales.

Effect of adding cholesterol-loaded cyclodextrin to thawed bovine sexed semen used for in vitro embryo production

\section{SUMMARY}

\section{ADDITIONAL KEYWORDS}

In vitro fertilization.

Sperm.

Cleavage.

Blastocyst.

INFORMATION

Cronología del artículo.

Recibido/Received: 14.10.2016

Aceptado/Accepted: 05.05 .2018

On-line: 15.07 .2018

Correspondencia a los autores/Contact e-mail:

ferre.luis@inta.gob.ar

\section{INTRODUCCIÓN}

La técnica de producción in vitro de embriones (PIVE) en bovinos es relativamente nueva en el mundo (fines de los 80) (First and Parrish 1987,Gordon and Lu 1990,Lu et al 1987). En los últimos años, el crecimiento de la PIVE ha sido notorio debido principalmente a 1) gran eficiencia en el uso del semen y 2) mayor cantidad de embriones producidos en comparación con otras técnicas reproductivas. La eficiencia en el uso de se- men se debe a que el proceso de fertilización se realiza micro-gotas lo cual genera un ahorro sustancial de semen (Greve and Madison 1991,Kato and Iritani 1993). La segunda ventaja comparativa tiene su fundamento en la posibilidad de colectar ovocitos de hembras con una alta frecuencia y en diversas condiciones fisiológicas (vacía, preñada, pre-púber) lo cual amplia enormemente el abanico de posibilidades en cuanto al avance genético y reducción del intervalo generacional (Kruip et al 1994,Looney et al 1994). 
Del $100 \%$ de ovocitos puestos a madurar, entre $80 \%$ y 90\% llegarán a Metafase II. De aquí el 70\%-80\% serán fertilizados y solo el 35\%-45\% llegarán al estadio de blastocisto en el día 7 de cultivo (Lonergan et al 2001). En caso de utilizar semen sexado, los porcentajes de fertilización y desarrollo embrionario son generalmente más bajos (Lu et al 1999,Zhang et al 2003). Esto se debería al efecto acumulado del proceso de sexado y posterior congelamiento/descongelamiento que los espermatozoides deben superar (Gosálvez et al 2011a, b,Rath et al 2009,Seidel and Garner 2002,Suh and Schenk 2003,Suh et al 2005).

La sobrevivencia de los espermatozoides de la mayoría de las especies de interés zootécnico al proceso de criopreservación está lejos de ser óptima (Barbas and Mascarenhas 2009,Medeiros et al 2002). Este problema se agrava si el semen es sometido al proceso de sexado previo a su congelación (Schenk et al 1999a). Está bien documentado el daño que puede ocurrir a los espermatozoides durante la criopreservación debido a factores tales como exposición a bajas temperaturas, estrés osmótico/mecánico, estrés oxidativo o una combinación de estos (Meyers 2005,Sieme et al 2015). A esto se le sumaría cambios perjudiciales en la estructura y funcionalidad de la membrana plasmática de los espermatozoides (Parks and Graham 1992,Silva and Gadella 2006).

El shock por frío que ocurre durante el proceso de congelación causa el cambio de fase de los lípidos y en consecuencia afecta la fluidez de membrana (Pegg 2015). La criopreservación induce el agotamiento o salida de colesterol de la membrana plasmática, que a su vez provoca su desestabilización (Akhoondi et al 2011,Amann and Hammerstedt 1993,Bailey et al 2000,Curry 2000,Sieme et al 2015). Además, la congelación puede desencadenar procesos capacitantes no fisiológicos en mayor o menor medida que podrían conducir a una reacción acrosómica prematura y ulterior pérdida de la funcionalidad y viabilidad de la gameta (Bailey et al 2000,Cormier et al 1997,Pons-Rejraji et al 2009).

Al problema del bajo número de espermatozoides que sobreviven a la congelación/descongelación se le suma efectos colaterales del proceso de sexado. En estudios realizados sobre espermatozoides de un mismo eyaculado (factorial: congelado vs. fresco y nosexado vs. sexado) revelaron que la congelación, y no el sexado, afecta negativamente la calidad del semen. No obstante, el proceso de sexado, y no la congelación, reduce la motilidad de los espermatozoides (Blondin et al 2009).

Como consecuencia del proceso de separación de espermatozoides portadores del cromosoma X o Y mediante citometría de flujo, la muestra seminal sexada muestra una marcada reducción de la motilidad postdescongelado y en la vida media de los espermatozoides (Blondin et al 2009,Schenk et al 1999b,Seidel 2007). Esto se debe a fuerzas de presión para su avance dentro del citómetro de flujo, efecto dilución, congelamiento y posterior descongelamiento, que provocan una serie de injurias acumuladas que afectan o comprometen las funciones espermáticas esenciales y su capacidad fecundante (Garner 2001, 2006,Gosálvez et al 2011a, b,Rath et al 2013,Seidel 2012). También fue sugerido que el proceso de sexado induciría reacciones precapacitantes generando subpoblaciones espermáticas en la muestra con estados de capacitación diferentes (Blondin et al 2009). Esto explicaría la necesidad de ajustar la concentración de factores capacitantes en el medio de fertilización in vitro de acuerdo al toro o muestra utilizada (Lu and Seidel 2004).

El agregado de ciclodextrinas cargadas con colesterol (CLC) aumentaría el contenido de colesterol del espermatozoide manteniéndose elevado aún luego de someterlos a un proceso de congelado y descongelado (Amorim et al 2009,Moce et al 2010a,Purdy and Graham 2004b). El tratamiento con CLC a los espermatozoides, previo a la congelación, ha demostrado mejorar la motilidad y viabilidad no sólo inmediatamente luego de post-descongelado sino también a lo largo del tiempo (Moce et al 2010b), aumentar el número de espermatozoides unidos a la zona pelúcida $(\mathrm{ZP})$ y porcentaje de penetración en ovocitos (Moore et al 2005,Purdy and Graham 2004a,Spizziri et al 2010) e incrementar la producción de embriones in vitro (Lee et al 2015).

El objetivo de este trabajo es estabilizar las membranas plasmáticas de espermatozoides sexados mediante el agregado de CLC post-descongelado y consecuentemente, prolongar su funcionalidad, aumentar su capacidad de fecundar ovocitos in vitro y producción de embriones.

\section{MATERIALES Y MÉTODOS}

Todos los químicos utilizados en este estudio se adquirieron de Sigma-Aldrich Chemical Co. (St. Louis, $\mathrm{MO}, \mathrm{USA}$ ) a menos que se indique lo contrario.

\section{SEMEN SEXADO}

Dosis de semen sexado congelado de tres toros fueron adquiridas de un proveedor comercial (Genex Cooperative, Inc., Shawano, WI, USA). Las dosis de semen de cada toro provienen del mismo lote/partida.

\section{DESCONGELACIÓN DEL SEMEN Y PROCESAMIENTO}

Las dosis de semen sexado se descongelaron a $37^{\circ}$ durante 45 segundos. Las dosis se secaron individualmente, el extremo sellado se cortó con un dispositivo aséptico y el contenido fue expulsado en un tubo de $1,5 \mathrm{ml}$ pre-calentado a $37^{\circ} \mathrm{C}$. El contenido de la dosis se mezcló bien e inmediatamente se procedió mejorar el semen mediante un gradiente de densidad: $40 \% / 80 \%$ (PureSperm, Spectrum Technologies, Healdsburg, CA, USA). La centrifugación se realizó a 700 g durante 15 minutos. Luego se descartó el sobrenadante, el pellet se resuspendió en TALP-Sperm ( $\mathrm{pH}=7.4,295 \mathrm{mOsm})$ (Parrish et al 1985,Parrish et al 1986) y se centrifugó nuevamente el tubo a $300 \mathrm{~g}$ por 5 minutos. La formulación final del TALP-Sperm fue: $100 \mathrm{mM} \mathrm{NaCl}, 3,1 \mathrm{mM}$ $\mathrm{KCl}, 2 \mathrm{mM} \mathrm{CaCl2}$, 0,3 mM NaH2PO4, 0,4 mM MgCl2, $25 \mathrm{mM}$ NaHCO3,10 mM HEPES, $1 \mathrm{mM}$ piruvato de sodio, 21,6 mM ácido láctico, $25 \mu \mathrm{g} / \mathrm{mL}$ gentamicina, y $6 \mathrm{mg} / \mathrm{mL}$ albúmina sérica bovina (BSA).

El pellet resultante de la segunda centrifugación fue resuspendido pipeteando suavemente en $100 \mu \mathrm{l}$ de 
medio sintético oviductal modificado SOF-FERT (Tervit et al 1972) e incubado a $38.5^{\circ} \mathrm{C}$ y $5 \% \mathrm{CO}_{2}$ en aire con humedad en saturación. La formulación final del SOFFERT consistió en: 107,7 mM NaCl, 7,16 mM KCl, 1,19 $\mathrm{mM} \mathrm{KH} \mathrm{PO}_{4}, 0,49 \mathrm{mM}, \mathrm{MgCl}_{2}, 1,17 \mathrm{mM} \mathrm{CaCl}_{2}, 5,3 \mathrm{mM}$ lactato de sodio, $25,07 \mathrm{mM} \mathrm{NaHCO}_{3}, 0,20 \mathrm{mM}$ piruvato de sodio, $0,5 \mathrm{mM}$ fructosa, $5 \mathrm{\mu g} / \mathrm{mL}$ gentamicina, 20 $\mathrm{\mu g} / \mathrm{mL}$ heparina, penicilamina $(3 \mu \mathrm{g} / \mathrm{mL})$, hipotaurina $(11 \mu \mathrm{g} / \mathrm{mL}$ ) y $6 \mathrm{mg} / \mathrm{mL}$ albúmina sérica bovina (BSA).

\section{ANÁLISIS DE SEMEN COMPUTARIZADO (CASA)}

La muestra de semen de cada toro fue analizada mediante un sistema CASA (SpermVision ${ }^{\circledR}$, Minitube, Alemania). La programación del CASA fue la siguiente: cuadros por segundo $=60$, número de cuadros $=30$; contraste mínimo $=50$, tamaño mínimo de la célula $=$ 5 ; tamaño de la célula $=5$, intensidad de la célula $=50$; velocidad de trayectoria $=50 \mu \mathrm{m} / \mathrm{s}$, rectitud $\geq 70 \%$, células lentas ( $\mu \mathrm{m} / \mathrm{s})$; velocidad media (VAP, $<30 \mu \mathrm{m} / \mathrm{s}$ ) y velocidad rectilínea (VSL, $<15 \mu \mathrm{m} / \mathrm{s}$ ). Las variables a medir con el sistema CASA fueron: porcentaje de motilidad total (MOT), porcentaje de motilidad progresiva (PMOT), VAP, VSL, velocidad curvilínea (VCL, um/s), amplitud de desplazamiento lateral de la cabeza (ALH, $\mu \mathrm{m})$, frecuencia de cruce (BCF, $\mathrm{Hz})$, índice de rectitud (STR, \%), índice de linealidad (LIN, \%) e índice de oscilación (WOB, \%). La temperatura de la cámara del CASA será de $37^{\circ} \mathrm{C}$. Se utilizó una cámara de 4 celdas Leja (IMV International Corp., Maple Grove, $\mathrm{MN}, \mathrm{USA}$ ) atemperada a $37^{\circ} \mathrm{C}$ para cargar la muestra de semen de acuerdo con las recomendaciones del fabricante. Teniendo en cuenta que los espermatozoides fueron teñidos previamente con Hoechst 33342 durante el proceso de sexado, se utilizó la óptica fluorescente IDENT del CASA. El número promedio de espermatozoides analizados por tratamiento fue de $344 \pm 78$ espermatozoides.

\section{Preparación de CLC}

La preparación de la CLC se realizó de acuerdo a una publicación pre-existente (Purdy and Graham 2004b). Inicialmente, se diluyó $1 \mathrm{~g}$ de metil- $\beta$ ciclodextrina en $2 \mathrm{ml}$ de metanol y $200 \mathrm{mg}$ de colesterol en $1 \mathrm{ml}$ de cloroformo. El stock colesterol se diluyó con metil- $\beta$-ciclodextrina hasta obtener una solución clara. Los disolventes fueron removidos mediante la incubación a $50^{\circ} \mathrm{C}$ durante 24 horas y los cristales formados fueron almacenados a temperatura ambiente. Momentos antes de su uso (30 min), $10 \mathrm{mg}$ de CLC fueron diluidos en $1 \mathrm{ml}$ de medio SOF-FERT e incubado en una incubadora a $38,5^{\circ} \mathrm{C}$ con un ambiente de $5 \% \mathrm{CO} 2$ y humedad en saturación. El tiempo de co-incubación del CLC y los espermatozoides sexados fue de $15 \mathrm{mi}-$ nutos antes de realizar el estudio CASA.

\section{DETERMINACIÓN DE LA CONCENTRACIÓN ESPERMÁTICA}

Se realizó una dilución 1:20 de la muestra de semen sexado con agua destilada $(5 \mu \mathrm{l}$ de semen $+95 \mu \mathrm{l}$ de agua destilada) en un micro-tubo. Luego de la homogeneización por pipeteo manual se procedió a preparar la cámara de Neubauer. Cada hemi-cámara fue llenada con $10 \mu \mathrm{l}$ de la dilución. Luego de 5 minutos de reposo se realizó el recuento mediante un microscopio a 400X. Se realizó el conteo de todos los espermatozoides del cuadrante central. El promedio de ambas cámaras fue multiplicado por 10000 y por x 20 (factor de dilución). El producto resultante es la cantidad de células espermáticas por $\mathrm{ml}$.

\section{DISEÑO EXPERIMENTAL}

Agregado de ClC a la muestra, eVAluAción con CASA Y FERTILIZACIÓN IN VITRO

La muestra de semen sexado fue inicialmente dividida en cuatro y aleatoriamente se le asignó una concentración de CLC $(\mathrm{O} \mathrm{mg}$ de CLC $=$ Control; $1,5 \mathrm{mg}$ de CLC; 3 mg de CLC y $6 \mathrm{mg}$ de CLC cada 120 millones de espermatozoides, respectivamente). Luego del agregado de CLC a cada grupo, la muestra tratada se incubó a $38,5^{\circ} \mathrm{C}$ con un ambiente de $5 \% \mathrm{CO} 2$ y humedad en saturación durante 15 minutos. El análisis con CASA comenzó inmediatamente después (tiempo 0), repitiéndose la evaluación a las 2, 4 y 8 hs. Las condiciones de incubación se mantuvieron constantes durante todo el período de muestreo. Se realizaron 3 repeticiones de cada nivel de CLC con cada uno de los cuatro toros.

Luego del análisis con CASA se procedió a la evaluación del semen sexado tratado con CLC en fertilización in vitro (FIV). El procedimiento de FIV fue el siguiente. Ovarios de matadero fueron transportados en un termo con solución fisiológica y antibióticos a $30-32^{\circ} \mathrm{C}$. Los folículos entre 2-6 $\mathrm{mm}$ de diámetro fueron aspirados y los complejos cúmulus-ovocito (COCs) compactos y cubiertos de manera completa por varias capas de células del cúmulus fueron seleccionados, lavados y madurados en grupos de 50 COCs en 400 $\mu 1$ de M199 suplementado con ALA-glutamina (0.1 $\mathrm{mM})$, piruvato sódico $(0.2 \mathrm{mM})$, gentamicina $(5 \mu \mathrm{g} /$ $\mathrm{ml})$, EGF (50 ng/ml), oFSH (50 ng/ml), bLH (3 ug/ $\mathrm{ml})$, cisteamina $(0.1 \mathrm{mM})$, y $10 \%$ suero fetal bovino (FBS) por 22-24 horas. La fertilización (Día 0) se llevó a cabo con semen sexado tratado con CLC $(0 \mathrm{mg}, 3 \mathrm{mg}$ y $6 \mathrm{mg}$ ) previamente mejorado por un gradiente de densidad. La dosis inseminante fue de $1 \times 106$. El medio de fertilización fue SOF-FERT suplementado con fructosa $(90 \mu \mathrm{g} / \mathrm{ml})$, penicilamina $(3 \mu \mathrm{g} / \mathrm{ml})$, hipotaurina (11 $\mu \mathrm{g} / \mathrm{ml}$ ) y heparina $(20 \mu \mathrm{g} / \mathrm{ml})$. Luego de 18 horas, los presuntos cigotos fueron denudados y cultivados en grupos de 15-20 en gotas de $50 \mu \mathrm{l}$ SOF-BSA (Holm et al 1999) por 7 días. En el día 3,5 se agregó 3\% de FBS. Las condiciones de incubación fueron de $38,5^{\circ} \mathrm{C}$ en un ambiente con $5 \%$ C02 y alta humedad durante la maduración, fertilización y cultivo. Además, durante el cultivo se utilizó baja tensión de oxígeno (5\%). En el día 2 de cultivo se evaluó el clivaje mientras que en el día 5,5 se evaluó la presencia de mórulas (MOR) y blastocistos tempranos (eBL). En el día 7 de cultivo se realizó el registro de blastocistos (BL) y blastocistos expandidos (BLEx) y en el día 9 la tasa de eclosión de la zona pelúcida. Se realizaron 3 repeticiones de cada tratamiento.

\section{ANÁLISIS ESTADÍSTICO}

Todos los análisis estadísticos se realizaron utilizando InfoStat version 2015 (Di Rienzo et al 2015) en conjunto con lenguaje R versión 3.2.3 (R Development Core Team 2015). Para considerar la totalidad del modelo experimental y las restricciones en la aleatoriza- 
Tabla I. Efecto de distintas concentraciones de CLC sobre la motilidad espermática (Effect of different concentrations of CLC on sperm motility).

\begin{tabular}{|c|c|c|c|c|}
\hline \multirow{2}{*}{ CASA } & \multicolumn{4}{|c|}{ CLC (mg) } \\
\hline & 0 & 1,5 & 3 & 6 \\
\hline MOT (\%) & $13,42 \pm 1,46^{A}$ & $13,17 \pm 1,41^{A}$ & $17,00 \pm 2,17^{\mathrm{B}}$ & $10,64 \pm 1,00^{A}$ \\
\hline PMOT (\%) & $11,58 \pm 1,61^{A B}$ & $8,87 \pm 1,08^{A}$ & $14,75 \pm 2,35^{\text {в }}$ & $8,84 \pm 1,07^{A}$ \\
\hline $\operatorname{VAP}(\mu \mathrm{m} / \mathrm{s})$ & $30 \pm 1,78^{A}$ & $51,76 \pm 3,61^{\mathrm{B}}$ & $47,77 \pm 3,2^{\mathrm{B}}$ & $27,06 \pm 1,58^{A}$ \\
\hline VSL $(\mu \mathrm{m} / \mathrm{s})$ & $26,13 \pm 1,79^{A}$ & $47,06 \pm 4,27^{B}$ & $43,52 \pm 3,76^{\mathrm{B}}$ & $23,57 \pm 1,58^{A}$ \\
\hline VCL $(\mu \mathrm{m} / \mathrm{s})$ & $54,38 \pm 3,77^{A}$ & $93,89 \pm 7,72^{\mathrm{C}}$ & $78,15 \pm 5,91^{\mathrm{B}}$ & $56,56 \pm 3,94^{A}$ \\
\hline LIN (\%) & $48,27 \pm 4,50^{\mathrm{B}}$ & $49,33 \pm 4,69^{\mathrm{B}}$ & $55,61 \pm 5,92^{c}$ & $43,27 \pm 3,65^{A}$ \\
\hline STR (\%) & $75,46 \pm 1,40^{A}$ & $86,49 \pm 1,82^{\mathrm{BC}}$ & $88,25 \pm 1,82^{\mathrm{B}}$ & $77,04 \pm 1,45^{\mathrm{A}}$ \\
\hline WOB (\%) & $55,45 \pm 4,04^{\mathrm{B}}$ & $54,43 \pm 3,90^{\mathrm{B}}$ & $61,20 \pm 4,87^{c}$ & $49,28 \pm 3,24^{A}$ \\
\hline $\mathrm{ALH}(\mu \mathrm{m})$ & $1,39 \pm 0,11^{\mathrm{A}}$ & $2,57 \pm 0,27^{\mathrm{B}}$ & $2,53 \pm 0,27^{\mathrm{B}}$ & $1,77 \pm 0,16^{\mathrm{AC}}$ \\
\hline $\mathrm{BCF}(\mathrm{Hz})$ & $16,46 \pm 1,19$ & $16,46 \pm 1,19$ & $16,46 \pm 1,19$ & $16,46 \pm 1,19$ \\
\hline
\end{tabular}

ción, los datos fueron analizados utilizando modelos generalizados mixtos independientes para cada variable de respuesta $(y)$, utilizando una distribución Gamma de parámetros $\kappa$ y $\lambda(1)$, predictor lineal $\eta_{\mathrm{ijk}}(2)$ y función de enlace canónico $g(\mu)=\mu^{-1}(3)$ :

$$
\begin{aligned}
& y \sim \Gamma(\kappa, \lambda) \\
& \eta_{i j k}=\mu+\alpha_{i}+\beta_{i}+\gamma_{i}+\alpha_{i} \times \gamma_{i}+\beta_{i} \times \gamma_{i}+b_{k} \\
& g(\mu)=1 / \mu=\eta_{i i k}
\end{aligned}
$$

donde la variable y (MOT, PMOT, VSL, VCL, WOB, LIN, STR, VAP, BCF y ALH) se modela a través de un predictor lineal $\left(\eta_{i j k}\right)$ en la que los efectos fijos se encuentran dados por la contribución de una media global $(\mu)$, el i-ésimo efecto de tiempo lineal $\left(\alpha_{\mathrm{i}}=0,2,4\right.$ y 8 horas) o cuadrático $\left(\beta_{\mathrm{i}}=\alpha_{\mathrm{i}}^{2}\right)$, la j-ésima concentración de CLC $\left(\gamma_{j}\right)$ y las correspondientes interacciones dobles de tiempo por CLC $\left(\alpha_{i} \times \gamma_{j}\right.$ y $\left.\beta_{i} \times \gamma_{j}\right)$, mientras que el único efecto aleatorio considera un intercepto para el k-ésimo efecto de toro $b_{k} \sim \mathrm{N}\left(0, \mathrm{I}_{\mathrm{b}}{ }^{2}\right)$, i. e., distribuido normal con media cero y varianza dada por la matriz identidad (I) multiplicada por el mismo parámetro $\sigma_{\mathrm{b}}{ }^{2}$. Se utilizó un nivel de significancia estadística de 0,05 para analizar los coeficientes del modelo. Para evaluar la diferencia entre los tratamientos de CLC se utilizó la prueba de menor diferencia significativa de Fisher (Fisher's Least Significant Difference) donde los pvalores fueron corregidos por Bonferroni. Los porcentajes de clivaje, producción de embriones y eclosión se analizaron mediante Chi-cuadrado de Pearson.

\section{RESULTADOS}

Los datos ajustados por el modelo con sus errores estándar para cada una de las variables analizadas en función de la concentración de CLC se muestran en la Tabla I. Se encontraron diferencias significativas $(\mathrm{P}<0,05)$ en MOT, PMOT, LIN y WOB para los espermatozoides sexados tratados con $3 \mathrm{mg}$ de CLC. Para el resto de las variables (VAP, VSL, VCL, STR y ALH) la concentración de 1,5 y 3 mg de CLC resultaron significativamente superiores a $6 \mathrm{mg}$ de CLC y al grupo control. La totalidad de los valores obtenidos para las diferentes variables de CASA a diferentes tiempos y concentraciones de CLC se presentan en la Tabla Suplementaria I.
Los valores de la tabla se expresan como medias \pm error estándar ajustados por el modelo correspondiente a cada variable de CASA. Resultados del test de la mínima diferencia significativa de Fisher son presentados como letras superíndice. Proporciones con diferente letras a lo largo de cada fila difieren significativamente (ajustado por Bonferroni $\mathrm{P}<0,05)$. Note que para la variable $\mathrm{BCF}$, no resultó estadísticamente significativo el efecto de concentración de CLC. Los valores esperados para las diferentes concentraciones de CLC han sido calculados considerando todos los tiempos de estudio $(0,2,4,6$ y 8 hrs.).

La motilidad disminuyó significativamente en relación al tiempo (Figura 1A). Cabe destacar que la concentración control (CLC $=0 \mathrm{mg}$ ) e incluso la de 1,5 mg de CLC presentan exactamente la misma evolución temporal en relación la variable de motilidad espermática (MOT). No obstante, la concentración de $3 \mathrm{mg}$ de CLC produce una reducción en la tasa de caída e incluso tiende a compensarse luego de 4 horas. Por el contrario, si se incrementa la concentración a $6 \mathrm{mg}$ de CLC, el efecto benéfico del CLC se torna contraproducente ya que presenta un desempeño inferior en comparación al control.

El resto de las variables CASA mostraron un comportamiento no lineal decreciente similar al mostrado para MOT en el Figura 1A, a excepción de LIN donde el comportamiento en la escala original alcanza un máximo para 4 horas (Figura 1B). El tratamiento de $3 \mathrm{mg}$ de CLC siempre presentó un intercepto no menor que el resto de las concentraciones y un mayor desempeño para todas las variables a excepción de VSL a 1,5 mg de CLC. Por otra parte, BCL presentó el mismo comportamiento independientemente de la concentración de CLC aplicada.

El clivaje de los cigotos fecundados con semen sexado tratado con CLC no mostro diferencias en 2 células a las 48 hrs. post-fertilización, mientras que si se registraron diferencias significativas en 4-8 células entre 3 y $6 \mathrm{mg}$ de CLC respecto al control (Tabla II). El clivaje total mostró diferencias significativas a favor de $3 \mathrm{mg}$ de CLC. El desarrollo embrionario posterior estuvo también marcado por una mayor velocidad de formación de $\mathrm{MOR} / \mathrm{eBL}$ a favor de los espermatozoides tratados con CLC en comparación grupo control. En los estadios embrionarios avanzados, el grupo $3 \mathrm{mg}$ de CLC mostró el mejor desempeño en producción de BL y embriones 
A

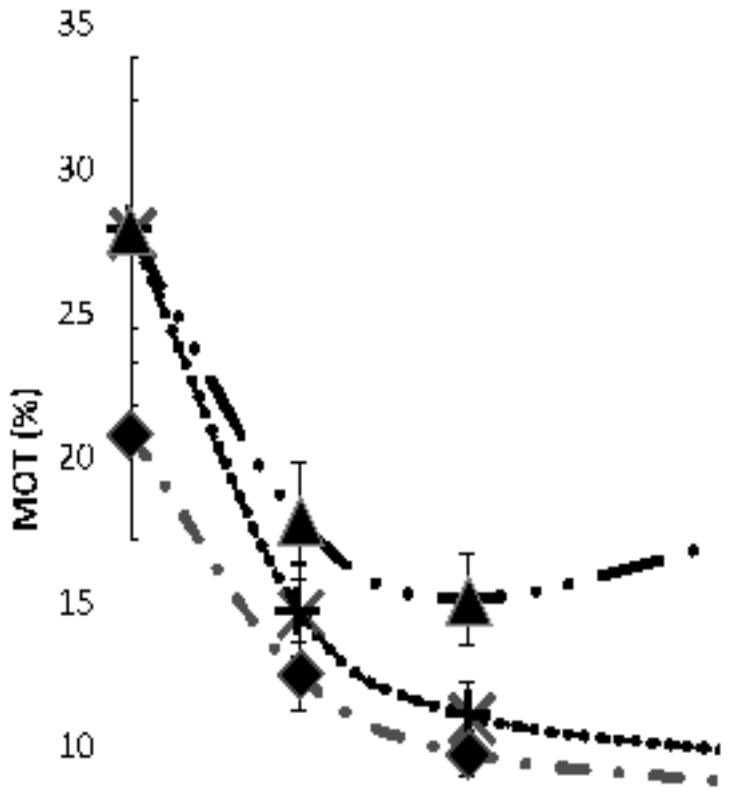

5

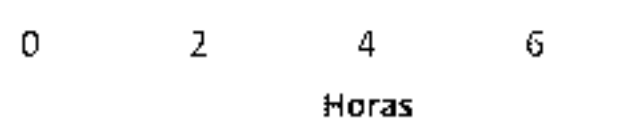

Horas

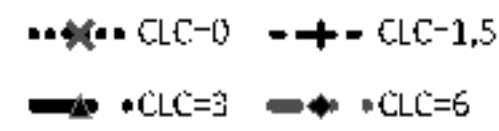

\begin{tabular}{ccccc}
\hline \multirow{2}{*}{ Horas } & \multicolumn{4}{c}{ CLC (mg) } \\
& 0 & 1,5 & 3 & 6 \\
\hline 0 & B & AB & AB & A \\
2 & B & B & B & A \\
4 & B & B & C & A \\
8 & AB & B & C & A
\end{tabular}

B

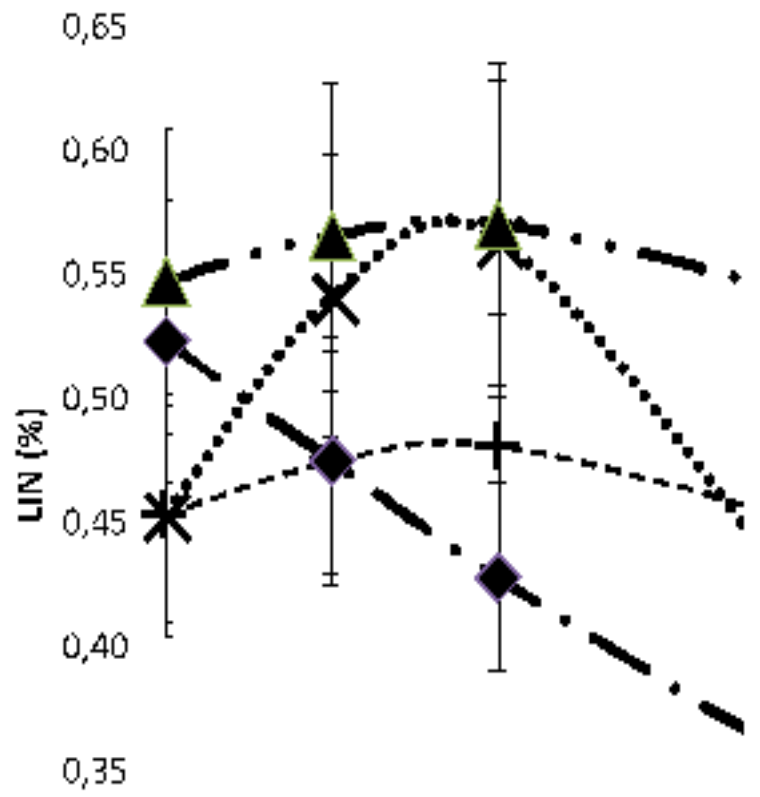

\begin{tabular}{ccccc}
\hline \multirow{2}{*}{ Horas } & \multicolumn{4}{c}{ CLC (mg) } \\
& 0 & 1,5 & 3 & 6 \\
\cline { 2 - 5 } 0 & A & AB & B & B \\
2 & BC & AB & C & A \\
4 & B & B & B & A \\
8 & B & C & C & A
\end{tabular}

Figura 1. Motilidad del semen sexado tratado con CLC a lo largo de tiempo. A) Motilidad (MOT). B) Motilidad Lineal (LIN). Las barras muestran el error estándar de la estimación y cada tabla muestra el resultado del LSD de Fisher (en letras A, B y C) para las diferentes concentraciones de CLC, para una hora fija, con corrección de Bonferroni con $(\alpha=0,05)($ Motility of semen sexing treated with CLC over time. Motility (MOT). Linear motility (LIN). The bars show the standard error of the estimation and each table shows the result of the Fisher LSD (in letters A, B and C) for the different concentrations of CLC, for a fixed time, with the correction of Bonferroni with $(\alpha=0.05)$.

totales. La eclosión de los blastocistos no mostro diferencia entre los tratamientos.

\section{DISCUSIÓN}

Durante el proceso de congelamiento/descongelado del semen, los espermatozoides son sometidos a procesos con cambios drásticos de condiciones internas y externas que pueden ejercer un acción perjudicial sobre el espermatozoide y causarle un daño temporal o permanente sobre su integridad y/o funcionalidad (Barbas and Mascarenhas 2009,Foote 2003a,Justice and Christensen 2013,Pegg 2015). Estas injurias sobre el espermatozoide podrían afectar su capacidad fecundante (Foote 2003b,Graham and Moce 2005). Una de las estructuras más afectada por el descenso de temperatura y los cambios osmóticos que se producen durante el proceso de congelación es la membrana plasmática. Los 
Tabla II. Fertilización y producción in vitro de embriones con semen sexado tratado con CLC (In vitro fertilization and production of embryos with sexing semen treated with CLC).

\begin{tabular}{|c|c|c|c|c|c|c|c|c|c|}
\hline \multirow{3}{*}{ CLC } & \multirow{3}{*}{$\begin{array}{l}\text { Ovocitos } \\
\text { (n) }\end{array}$} & \multirow{2}{*}{\multicolumn{2}{|c|}{ Clivaje (Día 2) }} & & \multicolumn{4}{|c|}{ Embriones } & \multirow{2}{*}{$\begin{array}{c}\text { Eclosión } \\
\text { Día } 9\end{array}$} \\
\hline & & & & & Día 5,5 & & Día 7 & & \\
\hline & & 2 C (\%) & $4-8$ C (\%) & Total $(\%)$ & MOR/eBL (\%) & $\mathrm{BL}(\%)$ & BLEx (\%) & Total (\%) & Total (\%) \\
\hline 0 mg CLC & 345 & $152(44 \%)$ & $72(21 \%)^{A}$ & $224(65 \%)^{A}$ & $117(34 \%)^{A}$ & $85(25 \%)^{\mathrm{AB}}$ & $23(7 \%)$ & $108(31 \%)^{A B}$ & $68(63 \%)$ \\
\hline 3 mg CLC & 370 & $179(48 \%)$ & $102(28 \%)^{\mathrm{B}}$ & $281(76 \%)^{\mathrm{B}}$ & $172(46 \%)^{\mathrm{B}}$ & $103(28 \%)^{A}$ & $35(9 \%)$ & $138(37 \%)^{\mathrm{A}}$ & $98(71 \%)$ \\
\hline 6 mg CLC & 364 & $155(43 \%)$ & $88(24 \%)^{\mathrm{AB}}$ & $243(67 \%)^{A}$ & $152(42 \%)^{\mathrm{B}}$ & $78(21 \%)^{\mathrm{B}}$ & $22(6 \%)$ & $100(27 \%)^{\mathrm{B}}$ & $61(61 \%)$ \\
\hline \multicolumn{10}{|c|}{$\begin{array}{l}\text { BL= Blastocisto } \\
\text { BLEx= Blastocisto expandido }\end{array}$} \\
\hline
\end{tabular}

cambios de organización lipídica que sufre la membrana son dependientes de la temperatura y se producen por la transición de fase líquida a gel de los fosfolípidos que la componen. El colesterol juega un papel muy importante en la regulación de estos fenómenos debido a que interactúa con los fosfolípidos alterando su comportamiento de cambio de fase (Parks and Graham 1992,Watson 2000). El proceso de sexado puede provocar efectos colaterales que también podrían afectar la viabilidad de los espermatozoides (Seidel and Garner 2002). Los espermatozoides sexados muestran una marcada reducción de la motilidad post-descongelado y un acortamiento en su vida media (Schenk et al $1999 b$ ). Esto se debe a fuerzas de presión para lograr su avance dentro del citómetro de flujo, efecto dilución, congelamiento y posterior descongelamiento, que provocan una serie de insultos acumulados que afectan o comprometen las funciones espermáticas esenciales y la capacidad fecundante (Garner 2001, 2006,Maxwell et al 2004). Los trabajos publicados muestran que el semen sexado produce resultados más bajos cuando lo comparamos con su homólogo no sexado (Blondin et al 2009,Lu et al 1999,Maxwell et al 2004,Wheeler et al 2006, Wilson et al 2005,Xu et al 2009,Zhang et al 2003). El mayor obstáculo para el semen sexado es la baja fertilidad (Amann 1999,Garner and Seidel Jr 2003,Seidel 2012,Suh et al 2005). Esta situación no pudo ni siquiera revertirse aumentando el número de espermatozoides (Dejarnette et al 2011,DeJarnette et al 2010). Las posibles causas de esta disminuida fertilidad serían una menor sobrevida post-descongelación, motilidad espermática, pre-capacitación e integridad acrosómica (Cran 2007,Frijters et al 2009,Gosálvez et al 2011a, b,Hollinshead et al 2003,Rath et al 2008,Schenk and Seidel 2007,Seidel 2007,Suh and Schenk 2003,Suh et al 2005). Se ha demostrado en varios estudios en otras especies que el tratamiento de los espermatozoides con CLC previo a la congelación mejora el porcentaje de espermatozoides que sobreviven al proceso en equinos (Combes et al 2000,Moore et al 2005,Zahn et al 2002), en bovinos (Moce and Graham 2006,Purdy and Graham 2004a), en ovinos (Moce et al 2010b) y caprinos (Konyali et al 2013). En algunas especies, el éxito en la adición de CLC depende de la temperatura y tiempo de incubación (Álvarez et al 2006). En el bovino, la temperatura de incubación $\left(37^{\circ} \mathrm{C}\right.$ o $\left.22^{\circ} \mathrm{C}\right)$ no afecta el efecto beneficioso del CLC y alargar la incubación de los espermatozoides con CLC más allá de los 15 minutos tampoco supera los resultados (Purdy and Graham 2004b). Estas diferencias encontradas entre especies pueden ser consecuencia de la mala elección del tipo de ciclodextrina, de una concentración de ciclodextrina inadecuada o puede que sean necesarios un periodo de incubación más largo o una temperatura de incubación más alta. Nuestros resultados muestran que la incubación de espermatozoides sexados con CLC mantiene la motilidad, motilidad progresiva y vigor durante un tiempo prolongado y que la capacidad fecundante de éstos espermatozoides es mayor que los no tratados. Estas observaciones son coincidentes con publicaciones realizadas con semen convencional las cuales muestran un aumento significativo de la motilidad, capacidad fecundante medida como la facultad de mantener la integridad acrosomal y desempeño en producción de embriones in vitro (Amorim et al 2009,Li et al 2006,Moce et al 2010a,Moce and Graham 2006,Moraes et al 2010,Purdy and Graham 2004a,Purdy and Graham 2004b). La eficiencia en la transferencia de colesterol de las ciclodextrinas depende, entre otros factores, de la duración de la exposición de la célula al complejo ciclodextrina-colesterol (Zidovetzki and Levitan 2007). El tiempo de incubación de 15 minutos con las CLC parecería ser suficiente si bien cabe remarcar que en el protocolo original, la adición e incubación de los espermatozoides con CLC se realiza antes de la congelación y los espermatozoides no experimentan el proceso de sexado. La concentración mayor de CLC utilizada en este estudio parecería causar daño y/o toxicidad ya que afectó los patrones de motilidad y producción de embriones in vitro. Cuando el contenido de colesterol es excesivo y aumenta por encima del nivel normal, éste se convierte en perjudicial para la supervivencia celular afectando la fluidez de membrana y su funcionalidad (Moce et al 2010a,Purdy and Graham 2004a,Purdy and Graham 2004b). La concentración óptima fue el tratamiento con $3 \mathrm{mg}$ de metil- $\beta$ ciclodextrina saturada de colesterol cada $120 \times 10^{6}$ espermatozoides sexados la que presentó mejores resultados en motilidad y desempeño in vitro. Esta cantidad de metil- $\beta$-ciclodextrina saturada de colesterol es similar a la utilizada en bovinos (1,5-3 mg /120x10 espermatozoides) (Purdy and Graham 2004a,Purdy and Graham 
2004b), en equinos (1,5 mg /120x10 espermatozoides) (Moore et al 2005) y en caprinos $\left(0,75-1,5 \mathrm{mg} / 120 \times 10^{6}\right.$ espermatozoides) (Barrera-Compean et al 2005). Los incrementos observados en los porcentajes de espermatozoides motiles fueron menores a los obtenidos en estudios realizados con semen convencional (Purdy and Graham 2004b). Tal vez se pueda aumentar y/o mantener aún más los patrones de motilidad ajustando mejor la adición de CLC mediante la determinación del contenido de colesterol en la membrana antes y después de la adición de CLC.

En conclusión, la incubación del semen sexado post-descongelado con CLC mantiene los patrones de motilidad durante más tiempo, aumenta la tasa de fecundación y producción de embriones in vitro.

\section{BIBLIOGRAFÍA}

Akhoondi M, HOldenhof, CStoll, HSieme, WFWolkers. 2011. Membrane hydraulic permeability changes during cooling of mammalian cells. Biochimica et Biophysica Acta (BBA)-Biomembranes 1808, 642-648. Álvarez AL, C Serres, P Torres, F Crespo, E Mateos, C Gómez-Cuétara. 2006. Effect of cholesterol-loaded cyclodextrin on the cryopreservation of donkey spermatozoa. Anim Reprod Sci 94, 89-91.

Amann RP. 1999. Issues affecting commercialization of sexed sperm. Theriogenology 52, 1441-1457.

Amann RP, RH Hammerstedt. 1993. In vitro evaluation of sperm quality: an opinion. Journal of andrology 14, 397-406.

Amorim EA, JK Graham, B Spizziri, M Meyers, CA Torres. 2009. Effect of cholesterol or cholesteryl conjugates on the cryosurvival of bull sperm. Cryobiology 58, 210-214.

Bailey JL, JF Bilodeau, N Cormier. 2000. Semen cryopreservation in domestic animals: a damaging and capacitating phenomenon. Journal of andrology 21, 1-7.

Barbas JP, RD Mascarenhas. 2009. Cryopreservation of domestic animal sperm cells. Cell and tissue banking 10, 49-62.

Barrera-Compean M, P Purdy, J Dzakuma, G Newton, L Nuti. 2005. Cholesterol-loaded cyclodextrin improves post-thaw goat sperm motility. Journal of animal science 83, 153.

Blondin P, M Beaulieu, V Fournier, N Morin, L Crawford, P Madan, WA King. 2009. Analysis of bovine sexed sperm for IVF from sorting to the embryo. Theriogenology 71, 30-38.

Combes GB, DD Varner, F Schroeder, RC Burghardt, TL Blanchard. 2000. Effect of cholesterol on the motility and plasma membrane integrity of frozen equine spermatozoa after thawing. Journal of reproduction and fertility, 127-132.

Cormier N, MA Sirard, JL Bailey. 1997. Premature capacitation of bovine spermatozoa is initiated by cryopreservation. Journal of andrology $18,461-468$.

Cran DG. 2007. XY sperm separation and use in artificial insemination and other ARTs. Society of Reproduction and Fertility supplement 65, 475-491.

Curry MR. 2000. Cryopreservation of semen from domestic livestock. Reviews of reproduction 5, 46-52.

Dejarnette JM, MA Leach, RLNebel, CEMarshall, CRMcCleary, JF Moreno. 2011. Effects of sex-sorting and sperm dosage on conception rates of Holstein heifers: is comparable fertility of sex-sorted and conventional semen plausible? Journal of dairy science 94, 3477-3483.

DeJarnette JM, CR McCleary, MA Leach, JF Moreno, RL Nebel, CE Marshall. 2010. Effects of 2.1 and $3.5 \times 10(6)$ sex-sorted sperm dosages on conception rates of Holstein cows and heifers. Journal of dairy science 93, 4079-4085.

Di Rienzo J, F Casanoves, M Balzarini, L Gonzalez, M Tablada, C Robledo 2015. InfoStat v2015. In InfoStat Group, College of Agricultural Sciences, National University of Córdoba, Argentina.
First NL, JJ Parrish. 1987. In-vitro fertilization of ruminants. Journal of reproduction and fertility 34, 151-165.

Foote RH. 2003a. Fertility estimation: a review of past experience and future prospects. Anim Reprod Sci 75, 119-139.

Foote RH. 2003b. Fertility estimation: a review of past experience and future prospects. Anim Reprod Sci 75, 119-139.

Frijters ACJ, E Mullaart, RMG Roelofs, RP van Hoorne, JF Moreno, O Moreno, JS Merton. 2009. What affects fertility of sexed bull semen more, low sperm dosage or the sorting process? Theriogenology 71, 64-67.

Garner DL. 2001. Sex-sorting mammalian sperm: concept to application in animals. Journal of andrology 22, 519-526.

Garner DL. 2006. Flow cytometric sexing of mammalian sperm. Theriogenology 65, 943-957.

Garner DL, GE Seidel Jr. 2003. Past, present and future perspectives on sexing sperm. Canadian Journal of Animal Science 83, 375-384.

Gordon I, KH Lu. 1990. Production of embryos in vitro and its-impact on livestock production. Theriogenology 33, 77-87.

Gosálvez J, MA Ramirez, C López-Fernández, F Crespo, KM Evans, ME Kjelland, JFMoreno. 201 la. Sex-sorted bovine spermatozoa and DNA damage: I. Static features. Theriogenology 75, 197-205.

Gosálvez J, MA Ramirez, C López-Fernández, F Crespo, KM Evans, ME Kjelland, JF Moreno. $2011 \mathrm{~b}$. Sex-sorted bovine spermatozoa and DNA damage: II. Dynamic features. Theriogenology 75, 206-21 1.

Graham JK, E Moce. 2005. Fertility evaluation of frozen/thawed semen. Theriogenology 64, 492-504.

Greve T, V Madison. 1991. In vitro fertilization in cattle: a review. Reproduction, nutrition, development 31, 147-157.

Holm P, PJ Booth, MH Schmidt, T Greve, H Callesen. 1999. High bovine blastocyst development in a static in vitro production system using SOFaa medium supplemented with sodium citrate and myo-inositol with or without serum-proteins. Theriogenology 52, 683-700.

Hollinshead FK, L Gillan, JK O'Brien, G Evans, WMC Maxwell. 2003. In vitro and in vivo assessment of functional capacity of flow cytometrically sorted ram spermatozoa after freezing and thawing. Reproduction, Fertility and Development 15, 351-359.

Justice T, GChristensen. 2013. Sperm cryopreservation methods. Methods in molecular biology 927, 209-215.

Kato H, A Iritani. 1993. In vitro fertilization in cattle. Mol Reprod Dev 36, 229-231.

Konyali C, C Tomas, E Blanch, E Gomez, J Graham, E Moce. 2013. Optimizing conditions for treating goat semen with cholesterol-loaded cyclodextrins prior to freezing to improve cryosurvival. Cryobiology 67, 124-131.

Kruip TA, R Boni, YA Wurth, MW Roelofsen, MC Pieterse. 1994. Potential use of ovum pick-up for embryo production and breeding in cattle. Theriogenology 42, 675-684.

Lee YS, S Lee, SH Lee, BK Yang, CK Park. 2015. Effect of cholesterolloaded-cyclodextrin on sperm viability and acrosome reaction in boar semen cryopreservation. Animal reproduction science 159, 124-130.

Li G, J Saenz, RA Godke, RV Devireddy. 2006. Effect of glycerol and cholesterol-loaded cyclodextrin on freezing-induced water loss in bovine spermatozoa. Reproduction 131, 875-886.

Lonergan P, D Rizos, F Ward, MP Boland. 2001. Factors influencing oocyte and embryo quality in cattle. Reproduction, nutrition, development 41, 427-437.

Looney CR, BR Lindsey, CL Gonseth, DL Johnson. 1994. Commercial aspects of oocyte retrieval and in vitro fertilization (IVF) for embryo production in problem cows. Theriogenology 41, 67-72.

Lu K, I Gordon, M Gallagher, H McGovern. 1987. Pregnancy established in cattle by transfer of embryos derived from in vitro fertilisation of oocytes matured in vitro. Veterinary Record 121, 259-260.

Lu KH, DG Cran, GE Seidel. 1999. In vitro fertilization with flowcytometrically-sorted bovine sperm. Theriogenology 52, 1393-1405.

Lu KH, GE Seidel, Jr. 2004. Effects of heparin and sperm concentration on cleavage and blastocyst development rates of bovine oocytes 
inseminated with flow cytometrically-sorted sperm. Theriogenology 62, 819-830.

Maxwell WMC, G Evans, FK Hollinshead, R Bathgate, SP de Graaf, BM Eriksson, L Gillan, KM Morton, JK O'Brien. 2004. Integration of sperm sexing technology into the ART toolbox. Anim Reprod Sci 82-83,79-95.

Medeiros CM, F Forell, AT Oliveira, JL Rodrigues. 2002. Current status of sperm cryopreservation: why isn't it better? Theriogenology 57, 327-344.

Meyers SA. 2005. Spermatozoal response to osmotic stress. Animal reproduction science 89, 57-64.

Moce E, E Blanch, C Tomas, JK Graham. 2010a. Use of cholesterol in sperm cryopreservation: present moment and perspectives to future. Reproduction in domestic animals = Zuchthygiene 45, 57-66.

MoceE, JK Graham. 2006. Cholesterol-loaded cyclodextrins added to fresh bull ejaculates improve sperm cryosurvival. J Anim Sci 84, 826-833.

MoceE, PHPurdy, JK Graham. 2010b. Treating ram sperm with cholesterolloaded cyclodextrins improves cryosurvival. Animal reproduction science 118, 236-247.

Moore Al, EL Squires, JK Graham. 2005. Adding cholesterol to the stallion sperm plasma membrane improves cryosurvival. Cryobiology 51, 241-249.

Moraes EA, JK Graham, CA Torres, M Meyers, B Spizziri. 2010. Delivering cholesterol or cholestanol to bull sperm membranes improves cryosurvival. Animal reproduction science 118, 148-154.

Parks J, J Graham. 1992. Effects of cryopreservation procedures on sperm membranes. Theriogenology 38, 209-222.

Parrish JJ, JL Susko-Parrish, NL First. 1985. Effect of heparin and chondroitin sulfate on the acrosome reaction and fertility of bovine sperm in vitro. Theriogenology 24, 537-549.

Parrish JJ, JL Susko-Parrish, ML Leibfried-Rutledge, ES Critser, WH Eyestone, NL First. 1986. Bovine in vitro fertilization with frozen-thawed semen. Theriogenology 25, 591-600.

Pegg DE. 2015. Principles of cryopreservation. Methods in molecular biology 1257, 3-19.

Pons-Rejraji H, JL Bailey, P Leclerc. 2009. Cryopreservation affects bovine sperm intracellular parameters associated with capacitation and acrosome exocytosis. Reproduction, fertility, and development 21, 525-537.

Purdy PH, JK Graham. 2004a. Effect of adding cholesterol to bull sperm membranes on sperm capacitation, the acrosome reaction, and fertility. Biology of reproduction 71, 522-527.

Purdy PH, JK Graham. 2004b. Effect of cholesterol-loaded cyclodextrin on the cryosurvival of bull sperm. Cryobiology 48, 36-45.

R Development Core Team 2015. R: A language and environment for statistical computing (Vienna, Austria, R Foundation for Statistical Computing).

Rath D, S Barcikowski, S de Graaf, W Garrels, R Grossfeld, S Klein, W Knabe, C Knorr, W Kues, H Meyer, J Michl, G Moench-Tegeder, C Rehbock, U Taylor, S Washausen. 2013. Sex selection of sperm in farm animals: status reportand developmental prospects. Reproduction 145, R15-R30.

Rath D, G Moench-Tegeder, U Taylor, LA Johnson. 2009. Improved quality of sex-sorted sperm: a prerequisite for wider commercial application. Theriogenology 71, 22-29.
Rath D, HJ Schuberth, P Coy, U Taylor. 2008. Sperm interactions from insemination to fertilization. Reproduction in domestic animals $=$ Zuchthygiene 43, 2-11.

SchenkJ, TSuh, D Cran, GSeidel. 1999a. Cryopreservation of flow-sorted bovine spermatozoa. Theriogenology 52, 1375-1391.

Schenk JL, GE Seidel, Jr. 2007. Pregnancy rates in cattle with cryopreserved sexed spermatozoa: effects of laser intensity, staining conditions and catalase. Society of Reproduction and Fertility supplement 64, 165-177.

Schenk JL, TK Suh, DG Cran, GE Seidel, Jr. 1999b. Cryopreservation of flow-sorted bovine spermatozoa. Theriogenology 52, 1375-1391.

Seidel GE, Jr. 2007. Overview of sexing sperm. Theriogenology 68, 443-446.

Seidel GE, Jr. 2012. Sexing mammalian sperm - Where do we go from here? The Journal of reproduction and development 58, 505-509.

Seidel GE, Jr., DL Garner. 2002. Current status of sexing mammalian spermatozoa. Reproduction 124, 733-743.

Sieme H, H Oldenhof, WF Wolkers. 2015. Sperm Membrane Behaviour during Cooling and Cryopreservation. Reproduction in Domestic Animals 50, 20-26.

Silva PF, BM Gadella. 2006. Detection of damage in mammalian sperm cells. Theriogenology 65, 958-978.

Spizziri B, M Fox, J Bruemmer, E Squires, J Graham. 2010. Cholesterolloaded-cyclodextrins and fertility potential of stallions spermatozoa. Animal Reproduction Science 118, 255-264.

Suh T, J Schenk. 2003. Pressure during flow sorting of bull sperm affects post-thaw motility characteristics. Theriogenology 59, 516.

Suh TK, JL Schenk, GE Seidel, Jr. 2005. High pressure flow cyłometric sorting damages sperm. Theriogenology 64, 1035-1048.

Tervit HR, DG Whittingham, LE Rowson. 1972. Successful culture in vitro of sheep and cattle ova. Journal of Reproduction and Fertility 30, 493-497.

Watson P. 2000. The causes of reduced fertility with cryopreserved semen. Animal Reproduction Science 60, 481-492.

Wheeler MB, JJ Rutledge, A Fischer-Brown, T VanEtten, S Malusky, DJ Beebe. 2006. Application of sexed semen technology to in vitro embryo production in cattle. Theriogenology 65, 219-227.

Wilson RD, KA Weigel, PM Fricke, JJ Rutledge, ML Leibfried-Rutledge, DL Matthews, VR Schutzkus. 2005. In vitro production of Holstein embryos using sex-sorted sperm and oocytes from selected cull cows. Journal of dairy science $88,776-782$.

Xu J, SA Chaubal, F Du. 2009. Optimizing IVF with sexed sperm in cattle. Theriogenology 71, 39-47.

Zahn FS, FO Papa, JJA Dell'Aqua. 2002. Cholesterol incorporation on equine sperm membrane: effects on post-thaw sperm parameters and fertility. Theriogenology 58, 237-240.

Zhang M, KH Lu, GE Seidel. 2003. Development of bovine embryos after in vitro fertilization of oocytes with flow cytometrically sorted, stained and unsorted sperm from different bulls. Theriogenology 60, 1657-1663.

Zidovetzki R, I Levitan. 2007. Use of cyclodextrins to manipulate plasma membrane cholesterol content: evidence, misconceptions and control strategies. Biochimica et biophysica acta 1768, 1311-1324. 